\title{
NUMERICAL AND STATISTICAL ANALYSIS OF ALIQUOT SEQUENCES
}

\author{
K. CHUM, R. K. GUY, M. J. JACOBSON, JR., AND A. S. MOSUNOV
}

\begin{abstract}
We present a variety of numerical data related to the growth of terms in aliquot sequences, iterations of the function $s(n)=\sigma(n)-n$. First, we compute the geometric mean of the ratio $s_{k}(n) / s_{k-1}(n)$ of $k$ th iterates for $n \leq 2^{37}$ and $k=1, \ldots, 10$. Second, we extend the computation of numbers not in the range of $s(n)$ (called untouchable) by Pollack and Pomerance 2016] to the bound of $2^{40}$ and use these data to compute the geometric mean of the ratio of consecutive terms limited to terms in the range of $s(n)$. Third, we give an algorithm to compute $k$-untouchable numbers $(k-1$ st iterates of $s(n)$ but not $k$ th iterates) along with some numerical data. Finally, inspired by earlier work of Devitt [1976], we estimate the growth rate of terms in aliquot sequences using a Markov chain model based on data extracted from thousands of sequences.
\end{abstract}

\section{INTRODUCTION}

An aliquot sequence is the iteration of the function $s(n)=\sigma(n)-n$, where $\sigma(n)$ is the sum of the divisors of $n$. Catalan [1888], corrected by Dickson 1913], conjectured that all aliquot sequences terminate. On the other hand, Guy and Selfridge 1975] conjectured that, starting with an even value of $n$, many, perhaps almost all, such sequences diverge. Aliquot sequences may terminate by reaching a prime $p$, since $s(p)=1$, by reaching a perfect number, for example $s(8128)=8128$, by reaching an amicable pair, for example $s(1184)=1210$ and $s(1210)=1184$, or by arriving in a longer cycle such as 14316, 19116, 31704, 47616, 83328, 177792, 295488, 629072, 589786, 294896, 358336, 418904, 366556, 274924, 275444, 243760, 376736, 381028, 285778, 152990, 122410, 97946, 48976, 45946, 22976, 22744, 19916, 17716, and then 14316 again.

Devitt [1976] used the average order of consecutive terms in a sequence, $s(n) / n$, taken over even values of $n$, namely $5 \pi^{2} / 24-1=1.0562$ as evidence that most aliquot sequences diverge, seemingly providing evidence in favor of Guy and Selfridge. However, it is the geometric mean, rather than the arithmetic mean, that is relevant here. Bosma and Kane 2012 have calculated this to be no bigger than $\mu=0.967 \ldots<1$, computed to 13 decimal digits $\lambda=\log \mu=-0.0332594808010 \ldots$ by Pomerance 2017]. Furthermore, Pomerance 2018] proved that the geometric mean of $s_{2}(n) / s(n)$, taken over even $n>2$, is also equal to $\mu$, where $s_{k}(n)$ denotes the $k$-th iterate of $s(n)$. These results favor Catalan and Dickson.

However, there is a possible flaw in this argument, namely that the range of $s(n)$ varies. Erdős [1973], Luca and Pomerance [2015], and Pollack et al. 2018] have

2010 Mathematics Subject Classification. Primary 11Y55; Secondary 11B83,11A25.

Key words and phrases. aliquot sequence, Guy-Selfridge conjecture, Markov chain.

The third author's research is supported by NSERC Discovery Grant RGPIN-2016-04545. 
obtained partial results about this. For example,

$$
s\left(192=2^{6} \cdot 3\right)=s\left(304=2^{4} \cdot 19\right)=s\left(344=2^{3} \cdot 43\right)=s\left(412=2^{2} \cdot 103\right)=316
$$

so that 316 should perhaps be given correspondingly more weight than values of $n$ not in the range of $s(n)$. These latter seem difficult to calculate (what are the solutions to $s(x)=n$ ?) but experimental evidence Pomerance and Yang 2014] suggests that as many as one-third of the even numbers are not in the range of $s(n)$.

As there are numbers not in the range of $s(n)$, aliquot sequences will tend to be tributary at the numbers that are in the range. Do these numbers, which should be counted by repetition, tend to be abundant (with $s(n)>n$ ) or deficient (with $s(n)<n$ )? Erdös [1973, 1976] and others have investigated this aspect. By focusing on numbers $n$ that are highly abundant, Erdös [1973] proved that there are infinitely many abundant numbers not in the range of $s(n)$. Note that a similar question about deficient numbers remains open. Further, Erdős improved on H. W. Lenstra's result that there are arbitrarily long increasing aliquot sequences Erdős 1976].

Another possible flaw in the argument of Bosma and Kane 2012 and Pomerance 2018 is that the function $s(n)$ tends to preserve certain divisibility properties of $n$. Guy and Selfridge [1975] explored the phenomenon of guides and drivers, particular divisors that tend to persist in consecutive sequence terms. Most of the more persistent drivers cause terms to be abundant. Pomerance [2017] has recently calculated the aliquot constants for terms with some divisibility restrictions, proving that $\log \mu$ is equal to $-0.3384354384114 \ldots,-0.2412950555350 \ldots$, and $0.1747760939329 \ldots$ when restricted to terms that are even and square-free, congruent to $2 \bmod 4$, and divisible by 4 , respectively. The facts that guides and drivers are almost all not square-free, and that 4 is itself a commonly-occurring guide, suggest that there may be a possibility that at least some aliquot sequences diverge.

In this work, we explored numerically two main lines of inquiry. The first was whether, in order to accurately capture the behavior of aliquot sequences, the geometric mean of $s(n) / n$ needs to take the variability of the range of $s(n)$ into account as opposed to being calculated over all even $n$, for example, as many of these values can never occur in a sequence. This can be studied in a number of ways. One possibility is to study properties of $k$ th iterates as in [Pomerance 2018], as the geometric mean of $s_{k}(n) / s_{k-1}(n)$ does indeed only take into account quantities in the range of $s(n)$. Another possibility is to enumerate explicitly touchable numbers (those that are in the range of $s(n)$ ) and use only these when computing the geometric mean of $s(n) / n$. Our second investigation was to account for the effects of guides and drivers by conducting numerical investigations measuring data occurring in actual sequences similar to Devitt [1976]; this method not only captures the variability of the range of $s(n)$, but also takes into account the influence of other factors such as guides and drivers Guy and Selfridge 1975] that the previous two approaches do not.

Our goal with this paper is to present novel numerical data on the growth of terms in aliquot sequences using all three of these approaches. First, in Section 2 we describe our computation of the geometric mean $\mu_{k}\left(X=2^{37}\right)$ of $s_{k}(n) / s_{k-1}(n)$ taken over all even $n \leq 2^{37}$ with $s_{k}(n)>0$ for $1 \leq k \leq 10$. In Section廿 we describe the enumeration of untouchable numbers (those not in the range of $s(n)$ ), extending the table of Pollack and Pomerance 2016] from a bound of $10^{10}$ to $2^{40}$. We use 
these data to compute $\mu_{1}\left(2^{40}\right)$ taken over even $n$ that are in the range of $s(n)$. We also present, in Section 4 an algorithm to compute $k$-untouchable numbers, those numbers in the range of $s_{k-1}(n)$ but not $s_{k}(n)$, and list all $k$-untouchable numbers up to $10^{7}$ for $k=2,3,4,5$ and to $10^{8}$ for $k=2,3$. Finally, in Section 6 we extend Devitt's work [Devitt 1976] by calculating the geometric mean of $s(n) / n$ obtained from 8000 randomly-selected aliquot sequences with initial terms of various sizes that were run until termination or until a term exceeded $2^{288}$.

Our data on $k$-th iterates show that $\mu_{k}\left(2^{37}\right)>1$ for $k \geq 6$, suggesting that terms with many successive preimages are more likely to increase. However, our data also show that $\mu_{k}(X)$ tends to decrease as the bound $X$ increases. In view of Pomerance's conditional result stated in Pomerance 2018, Theorem 2.4], it is natural to conjecture that $\mu_{k}(X)$ approaches $\mu$ for all $k$. On the other hand, the empirical estimate we obtain by extending Devitt's work is greater than one, suggesting that further extensions to the analytic results of Bosma and Kane 2012] and Pomerance 2018, 2017] that also account for the effect of guides and drivers would be of great interest.

\section{Geometric Means of $k$ th Iterates}

Bosma and Kane 2012] proved that the geometric mean of $s(n) / n$ taken over even $n$ is equal to $\mu=0.967 \ldots<1$, and

$$
\log \mu=-0.0332594808010 \ldots
$$

was computed to 13 decimal digits of accuracy by Pomerance 2017]. Further, Pomerance 2018] proved that the geometric mean of $s_{2}(n) / s(n)$, taken over even $n>2$, is also equal to $\mu$. Both of these results give strong probabilistic evidence that most aliquot sequences converge.

A natural question is whether the same mean also holds for $k$ th iterates, where $k>2$. In some sense, considering these quantities for larger values of $k$ might give a more accurate picture of the average behavior of aliquot sequences, as the ratio of successive terms is being measured further along in a sequence as opposed to the first two or three terms. Motivated by this question, we performed some numerical computations of the quantity

$$
\lambda_{k}(X)=\frac{1}{\# B_{k}(X)} \sum_{n \in B_{k}(X)} \log \frac{s_{k}(n)}{s_{k-1}(n)},
$$

where

$$
B_{k}(X)=\left\{n \in \mathbb{N}: n \leq X, n \text { is even and } s_{k}(n)>0\right\} .
$$

To evaluate $\lambda_{k}(X)$, we start by computing $\sigma(n)$ for all $n$ such that $1 \leq n \leq X$ and storing the resulting values into a lookup table $\Sigma$. This was done by using the algorithm of Moews and Moews [1991]. For each even $n$, we compute the value $s(n)=\sigma(n)-n$ and store it into one of the sets $\mathcal{S}_{1}$ or $\mathcal{L}_{1}$, depending on whether $s(n) \leq X$ or not. We compute $\lambda_{1}(X)$ directly from the values stored in $\mathcal{S}_{1}$ and $\mathcal{L}_{1}$.

In order to compute $\lambda_{k}(X)$ for $k>1$, we need to determine $s_{k}(n)=s\left(s_{k-1}(n)\right)$ for all even $n \leq X$. To do so, for each $m$ in $\mathcal{S}_{k-1}$ we find $\sigma(m)$ in our lookup table $\Sigma$. In order to determine $\sigma(m)$ for all $m$ in $\mathcal{L}_{k-1}$, we factor $m$ directly. In our implementation, several values of $m$ in $\mathcal{L}_{k-1}$ are factored in parallel. Given all the $\sigma(m)$ values, we compute $s(m)=\sigma(m)-m$ and, in the case that $s(m) \neq 0$, store it in either $\mathcal{S}_{k}$ or $\mathcal{L}_{k}$, depending on whether $s(m) \leq X$ or not. From these data, we 
obtain $s(m) / m=s_{k}(n) / s_{k-1}(n)$ for some even number $n$ such that $s_{k-1}(n)=m$, and the resulting values will range over all even $n \leq X$ with $s_{k}(n)>0$. Finally, we compute $\lambda_{k}(X)$ by iterating over $\mathcal{S}_{k}$ and $\mathcal{L}_{k}$. At this point, we can discard the sets $\mathcal{S}_{k-1}$ and $\mathcal{L}_{k-1}$ and use the sets $\mathcal{S}_{k}$ and $\mathcal{L}_{k}$ in conjunction with the procedure described previously to compute $\lambda_{k+1}(X)$.

Since $\sigma(n)$ for $1 \leq n \leq 2^{37}$ fits into unsigned long ( 8 bytes), the size of the table $\Sigma$ reaches $1 \mathrm{~TB}$ in size, while the tables $\mathcal{S}_{1}$ and $\mathcal{L}_{1}$ reach $512 \mathrm{~GB}$. None of those can fit into memory, and so the calculations were handled by partitioning our data into several files, storing the files into the hard disk, and loading them one-by-one into the memory.

All the computations described in this section, as well as in Sections 3 and 4 . were carried on WestGrid's supercomputer Hungabee, located at the University of Alberta [WestGrid 2017], Canada. Hungabee is a 16 TB shared memory system with 2048 Intel Xeon cores, 2.67GHz each. Each user of Hungabee may request at most $8 \mathrm{~GB}$ of memory per core. Also, Hungabee provides a high performance 53 TB storage space, which allows us to write to multiple disks in parallel. All the data, as well as the programs used for its computation, are available from the authors upon request.

Define $s_{0}(n):=n$. Using Hungabee, we evaluated $\lambda_{k}(X)$ for $k=1,2, \ldots, 10$ and $X=2^{15}, 2^{16}, \ldots, 2^{37}$ (see Table 10). The timings of our computations for $X=2^{37}$ are as follows. The computation of $\lambda_{k}(X)$ for $k=1,2, \ldots, 10$ was mostly done sequentially, processing each of 1024 files individually, and using 128 processors in parallel solely for the purpose of factoring numbers that are larger than $2^{37}$. The computation of 1024 files storing $\sigma(n)$ for all $n \leq 2^{37}$ took $4 \mathrm{~h} 42 \mathrm{~m}$ 8s of real time, using 256 processors (total CPU time $7 \mathrm{w} 1 \mathrm{~d} 3 \mathrm{~h} 46 \mathrm{~m} 8 \mathrm{~s}$ ). With the table of $\sigma(n)$ stored in memory, the evaluation of $\lambda_{1}(X)$ took $3 \mathrm{~h} 4 \mathrm{~m} 39 \mathrm{~s}$ of total CPU time. No parallel computations were needed in this case. The computation of a single value $\lambda_{k}(X)$ for $k \geq 2$ requires approximately 4 times more, as the computation of $s\left(s_{k-1}(n)\right)$ is not as straightforward, and when $s_{k-1}(n)>2^{37}$ it must be factored directly in order to compute $\sigma\left(s_{k-1}(n)\right)$. The computation of $\lambda_{k}(X)$ for

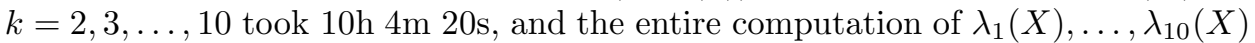
for $X=2^{37}$ took $3 \mathrm{~d} 21 \mathrm{~h} 43 \mathrm{~m} 38$ s of real time using 128 processors. It is difficult to estimate the total CPU time due to the fact that most of the program is sequential, and only factorization of large numbers is carried in parallel.

We report that the geometric means $\mu_{k}(X)=e^{\lambda_{k}(X)}$ exceed 1 for $X=2^{37}$ and $k=6,7,8,9,10$ when averaged over all even $n$ such that $s_{k}(n)>0$. Moreover, as $k$ increases, the geometric means grow, too. As the function $\lambda_{k}$ pre-selects those $n$ that have not yet reached a prime, it is not surprising that as $k$ increases, so does $\lambda_{k}(X)$. What is more interesting is that, as $k$ remains fixed, the geometric means decrease with the growth of $X$, possibly approaching the geometric mean of $s(n) / n$.

Figure 11 depicts $\lambda_{k}\left(2^{r}\right)$ as $r$ varies from 15 to 37 and $k$ remains fixed. The red line corresponds to $\lambda_{1}\left(2^{r}\right)$, the green line to $\lambda_{2}\left(2^{r}\right)$, and the blue lines, from bottom to top, correspond to $\lambda_{3}\left(2^{r}\right), \lambda_{4}\left(2^{r}\right), \ldots, \lambda_{10}\left(2^{r}\right)$. As the value of $X$ approaches infinity, the functions $\lambda_{1}(X)$ and $\lambda_{2}(X)$ converge to $\log \mu \approx-0.033$ from below. The convergence of $\lambda_{1}(X)$ is almost immediate and can hardly be observed. The convergence of $\lambda_{2}(X)$ is rather slow, and this was explained theoretically in Pomerance 2018]. As is seen in Figure 1, the values of $\lambda_{k}(X)$ for $k=3,4, \ldots, 10$, 


\begin{tabular}{|c||c|c|c|c|c|c|}
\hline$X$ & $2^{15}$ & $2^{20}$ & $2^{25}$ & $2^{30}$ & $2^{35}$ & $2^{37}$ \\
\hline \hline$\lambda_{1}(X)$ & -0.03336 & -0.03326 & -0.03326 & -0.03326 & -0.03326 & -0.03326 \\
\hline$\lambda_{2}(X)$ & -0.09523 & -0.05338 & -0.04273 & -0.03910 & -0.03749 & -0.03706 \\
\hline$\lambda_{3}(X)$ & -0.06434 & -0.01980 & -0.01399 & -0.01531 & -0.01763 & -0.01849 \\
\hline$\lambda_{4}(X)$ & -0.07394 & -0.01333 & -0.00568 & -0.00752 & -0.01081 & -0.01205 \\
\hline$\lambda_{5}(X)$ & -0.07451 & -0.00830 & 0.00296 & 0.00132 & -0.00259 & -0.00411 \\
\hline$\lambda_{6}(X)$ & -0.08764 & -0.00536 & 0.01496 & 0.00772 & 0.00322 & 0.00145 \\
\hline$\lambda_{7}(X)$ & -0.09902 & -0.00053 & 0.01655 & 0.01478 & 0.00977 & 0.00779 \\
\hline$\lambda_{8}(X)$ & -0.10820 & 0.00033 & 0.02212 & 0.02059 & 0.01515 & 0.01297 \\
\hline$\lambda_{9}(X)$ & -0.11139 & 0.00316 & 0.02822 & 0.02669 & 0.02091 & 0.01854 \\
\hline$\lambda_{10}(X)$ & -0.11341 & 0.00708 & 0.032823 & 0.03193 & 0.02592 & 0.02339 \\
\hline
\end{tabular}

TABLE 1. Values of $\lambda_{k}(X)$.

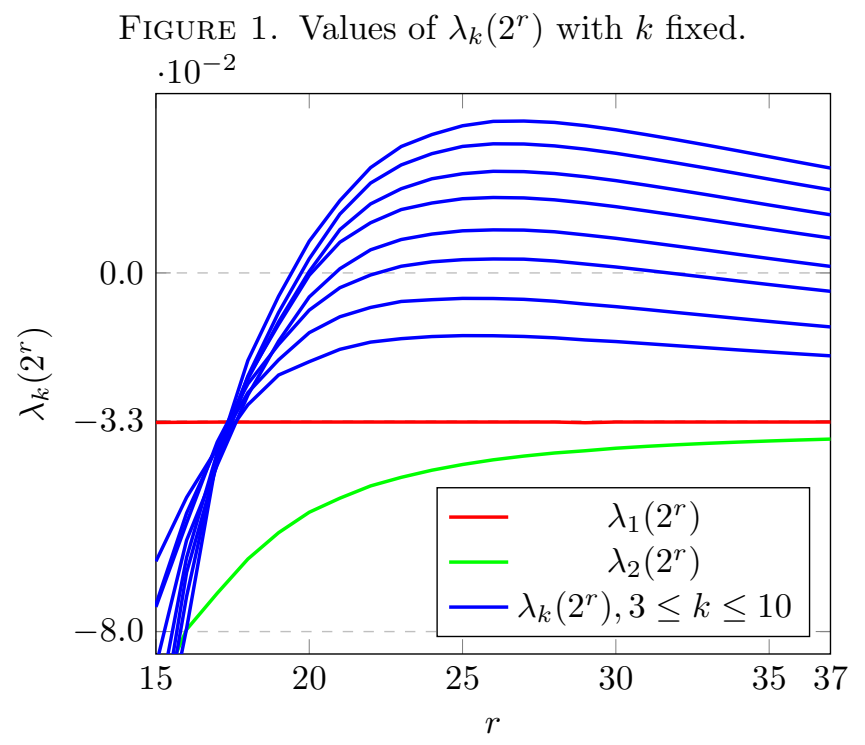

unlike those for $k=1,2$, are strictly decreasing. We also see quite a remarkable difference between the behavior of $\lambda_{1}(X), \lambda_{2}(X)$ and $\lambda_{k}(X)$ for $k \geq 3$.

Let

$$
s^{-1}(n):=\{m \in \mathbb{N}: s(m)=n\} .
$$

It was conjectured by Erdős, Granville, Pomerance, and Spiro see Pomerance 2018, Conjecture 2.3] that, if $A$ is a set of natural numbers of asymptotic density 0 , then $s^{-1}(A)$ also has asymptotic density 0 . Assuming this conjecture, Pomerance proved that for each integer $k \geq 2$ there is a subset $A_{k} \subseteq \mathbb{N}$ of asymptotic density 1 with respect to $\mathbb{N}$ such that the geometric mean of $s_{k}(n) / s_{k-1}(n)$ averaged over all $n \in A_{k}$ converges to the Bosma-Kane constant $\mu$ Pomerance 2018, Theorem 2.4]. Our computations serve as evidence of Pomerance's conditional result with $2 \mathbb{N}$ in place of $\mathbb{N}$. 
Finally, we have also evaluated $\lambda_{1}(X)$ up to $X=2^{40}$ in order to compare the value $-0.0332594808010 \ldots$ computed to 13 decimal digits of accuracy by Pomerance [2017]. To 13 decimal places, we get $\lambda_{1}\left(2^{40}\right)=-0.0332594808048 \ldots$, matching Pomerance's computed value to the first 11 digits.

\section{Tabulation of Untouchable Numbers}

A number $n$ is called untouchable if there is no $m$ such that $n=s(m)$. It is called touchable otherwise. According to a slightly stronger variant of Goldbach's conjecture, for every odd integer $n \geq 9$ there exist distinct prime numbers $p$ and $q$ such that

$$
n=1+p+q=s(p q) .
$$

The fact that 5 is the only odd untouchable number follows from this conjecture, since $1=s(2), 3=s(4)$ and $7=s(8)$, but no such expression exists for $n=5$. This variant of Goldbach's conjecture has been verified computationally by Oliveira e Silva to $4 \cdot 10^{18}$ Oliveira e Silva 2013].

Pollack and Pomerance 2016] gave a heuristic justification that the set of untouchable numbers has natural asymptotic density equal to

$$
\Delta=\lim _{X \rightarrow \infty} \Delta(X)
$$

where

$$
\Delta(X)=\frac{1}{\log X} \sum_{\substack{n \leq X \\ 2 \mid n}} \frac{1}{n} e^{-n / s(n)} .
$$

In order to verify the conjecture of Pollack and Pomerance computationally, we tabulated all even untouchable numbers to $2^{40}$. Previously, the tabulation of untouchable numbers was done by Pomerance and Yang 2014] to $10^{8}$ and by Pollack and Pomerance [2016] to $10^{10}$. In all three cases the tabulation was done using the algorithm of Pomerance and Yang described in [Pomerance and Yang 2014]. However, our computations were carried on a much larger scale, requiring some additional techniques, such as buffering and the usage of the hard disk. In the end, our counts of untouchable numbers to $10^{10}$ matched those given by Pollack and Pomerance [2016]. The fourth author is grateful to Prof. Pollack for his help to verify the correctness of our computations.

Our modification of the algorithm of Pomerance and Yang is as follows. In order to tabulate all untouchable numbers up to $X$, we determine all touchable numbers first, and then count the numbers that are left out. We use a bit array to represent each integer less than $X$, initialized to zero and split over $K$ files. We precompute $\sigma(n)$ for all odd $n$ in the range from 1 to $X / 2$ using the algorithm of Moews and Moews [1991]. Then we use the algorithm of Pomerance and Yang to tabulate all touchable numbers in parallel. Each touchable number $m$ found is stored in one of $K$ buffers, each of which holds integers represented in a particular binary file. When a buffer is full, the bit in the corresponding file that represents each integer stored in the buffer is set to one. At the end, the zero bits in each file correspond to untouchable numbers.

Our computations were done in parallel, using 8 processors, with 1 thread per processor. In order to maintain a reasonable balance between the total number of files and the size of an individual file, the number of files was chosen to be equal to 4096. With this setup, the program for tabulation of untouchable numbers to 
$X=2^{40}$ terminated in $3 \mathrm{~d} 4 \mathrm{~h} 7 \mathrm{~m} 11 \mathrm{~s}$ (the total CPU time is $3 \mathrm{w} 4 \mathrm{~d} 8 \mathrm{~h} 57 \mathrm{~m} 28 \mathrm{~s}$ ). In order to reduce the number of disk I/O operations, as well as to reduce the amount of storage space required, we compressed our data by fitting the information on the type of every even number $n$ into 2 bits, depending on whether the equation $n=s(m)$ has odd/even solutions, or both, or no solutions at all. Thus, for example, the number 2 has type 00, since it is untouchable; number $4=s(9)$ has type 01; number $10=s(14)$ has type 10 ; and number $6=s(6)=s(25)$ has type 11 . The size of our data is $128 \mathrm{~GB}$.

\begin{tabular}{|r|r|r|r|r|}
\hline$X$ & $U(X)$ & $U(X) / X$ & $\Delta(X)$ & $\Delta(X)-U(X) / X$ \\
\hline \hline $10^{4}$ & 1212 & 0.121200 & 0.161059 & 0.039859 \\
\hline $10^{5}$ & 13863 & 0.138630 & 0.164577 & 0.025947 \\
\hline $10^{6}$ & 150232 & 0.150232 & 0.166923 & 0.016691 \\
\hline $10^{7}$ & 1574973 & 0.157497 & 0.168599 & 0.011102 \\
\hline $10^{8}$ & 16246940 & 0.162469 & 0.169857 & 0.007388 \\
\hline $10^{9}$ & 165826606 & 0.165827 & 0.170834 & 0.005007 \\
\hline $10^{10}$ & 1681871718 & 0.168187 & 0.171617 & 0.003430 \\
\hline $10^{11}$ & 16988116409 & 0.169881 & 0.172257 & 0.002376 \\
\hline $10^{12}$ & 171128671374 & 0.171129 & 0.172790 & 0.001661 \\
\hline $2^{40}$ & 188206399403 & 0.171173 & 0.172810 & 0.001637 \\
\hline
\end{tabular}

TABLE 2. Counts of untouchable numbers to $2^{40}$.

The counts are given in Table 2 By $U(X)$ we denote the total count of untouchable numbers up to $X$. Our computations seem to suggest that the natural asymptotic density of the set of untouchable numbers exists. Looking back at (3.1), for $X=2^{40}$ the expression inside the limit has value 0.17281 , and this is quite close to our value $U\left(2^{40}\right) / 2^{40} \approx 0.17117$. The values of $\Delta(X)$ for different $X$ are also given in Table 2. Figure 2 depicts the graphs of $U(X) / X$ and $\Delta(X)$. The heuristics of Pollack and Pomerance suggests that the graphs of $U(X) / X$ and $\Delta(X)$ should approach the same limiting value, and according to Figure 2 this certainly seems to be the case.

It was suggested by the second author, as well as by Carl Pomerance, to consider different variants of the quantity $\lambda_{1}(X)$ by replacing the set $B_{1}(X)$ with something else. For example, averaging over the set of all even touchable numbers, rather than over all even numbers, would be more appropriate, as the untouchable numbers never really occur in aliquot sequences and therefore do not affect their behavior. According to Table 2, over a third of all even numbers up to $2^{40}$ are untouchable, and so they might influence the resulting quantity $\mu_{1}(X)=\exp \left(\lambda_{1}(X)\right)$ quite significantly. We report that the geometric mean of $s(n) / n$ taken over touchable even $n \leq 2^{40}$ is equal to 0.915285 , which is less than $\mu \approx 0.967$. On the other hand, the geometric mean of $s(n) / n$ taken over untouchable even $n \leq 2^{40}$ is equal to 1.076. Thus the computations suggest that the presence of untouchable numbers actually forces the geometric mean over all even numbers to increase rather than to decrease.

Heuristically speaking, it is perhaps not so surprising that even numbers in the range tend to be somewhat less abundant than even numbers in the domain. Since any function $f(n)$ with $f(n)$ large compared with $n$ will miss many numbers, we do 
FiguRe 2. Values of $U\left(2^{r}\right) / 2^{r}$ and $\Delta\left(2^{r}\right)$ for $1 \leq r \leq 40$.

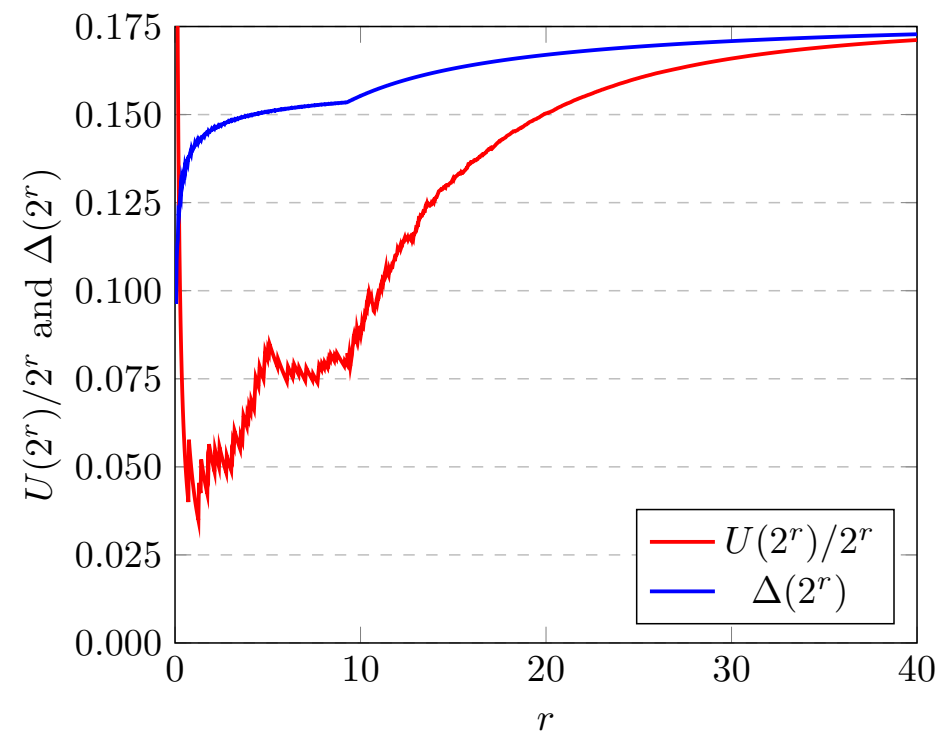

expect many $n$ not to appear in the range of $s(n)$ when $s(n)$ is significantly larger than $n$. In fact, this observation lies in the core of the argument of Erdös [1973].

Another variant of $\lambda_{1}(X)$ that we considered incorporates the fact that certain numbers have more preimages under $s(n)$ than others, and so they could be influencing the behavior of aliquot sequences more. For example, the number 160154 has 15 preimages under $s(n)$, namely

$$
\begin{array}{rlllll}
160154 & =s(152776) & =s(183016) & =s(251614) & =s(260182) & =s(296074) \\
& =s(298234) & =s(302842) & =s(310942) & =s(311086) & =s(312166) \\
& =s(313822) & =s(313966) & =s(315082) & =s(315226) & =s(315262) .
\end{array}
$$

Hence the value of $\log (s(n) / n)$ for $n=160154$ should perhaps be considered with the weight 15 instead of weight 1 as it was done in the computation of $\lambda_{1}(X)$. Clearly, every untouchable number in this case would have weight 0 . A slight modification of our program for the tabulation of untouchable numbers allowed us to tabulate the values of $\# s^{-1}(n)$ for all even $n$ up to $X=2^{40}$, and evaluate the quantity

$$
\tilde{\lambda}(X)=\left(\sum_{\substack{n \leq X \\ 2 \mid n}} \# s^{-1}(n)\right)^{-1} \sum_{\substack{n \leq X \\ 2 \mid n}} \# s^{-1}(n) \log \frac{s(n)}{n} .
$$

Once again, our computations were done in parallel, using 64 processors with one thread per processor. The number of files was chosen to be equal to 8192 . With this setup, the program for the tabulation of $\# s^{-1}(n)$ for even $n$ up to $X=2^{40}$ terminated in $1 \mathrm{w} 8 \mathrm{~h} 34 \mathrm{~m} 37 \mathrm{~s}$ (the total CPU time is $1 \mathrm{y} 42 \mathrm{w} 3 \mathrm{~d} 20 \mathrm{~h} 55 \mathrm{~m} \mathrm{28s}$ ). Evidently, in comparison to the tabulation of untouchable numbers, the tabulation of $\# s^{-1}(n)$ took significantly more time. The main reason for the degradation in performance lies in the fact that we recorded not the type of a number, but the 
actual count of the total number of preimages. While the type of a number fits into 2 bits, the information on the number of preimages fits into 8 bits 1 so the total number of disk I/O operations increased at least 4 times. The total size of the data is $512 \mathrm{~GB}$.

We report that for $X=2^{40}$ the geometric mean $\exp (\tilde{\lambda}(X))$ is equal to 0.862346 , which is less than the Bosma-Kane constant $\mu$. In fact, it is even less than the geometric mean taken over touchable even numbers up to $X$.

\section{Tabulation of $k$-Untouchable Numbers}

Let $k$ be a positive integer exceeding one. A $k$-untouchable number is a number lying in the range of $s_{k-1}$, but not in the range of $s_{k}$. Just like untouchable numbers, $k$-untouchable numbers do not appear in aliquot sequences $\left\{s_{i}(n)\right\}_{i=0}^{\infty}$ for $i \geq k$, so they can also be disregarded when computing the geometric mean of $s(n) / n$.

In this section, we introduce an algorithm which tabulates all $k$-untouchable numbers up to $X$ and $k \leq K$. We use this algorithm to tabulate $k$-untouchable numbers to $10^{7}$ for $k=2,3,4,5$ and to $10^{8}$ for $k=2,3$, and then use these data to compute the geometric mean of $s(n) / n$ over all even $n \leq X$ that are not $k$ untouchable for various values of $k$ and $X$.

Our computations were done as follows. First we precomputed the table of preimages for all even $n \leq X$ using the algorithm of Pomerance and Yang (here $X$ was chosen to be $2^{30}$ ). Then for each even $n$ we launched the recursive procedure described below (Algorithm 10 to search for preimages under $s_{k}(n)$. This procedure terminates if we find some even $m>X$ such that $n=s_{i}(m)$ with $i<k$. Our experimental observations suggest that when preimages for all even $n \leq X$ are precomputed, the algorithm produces all $k$-untouchable numbers up to $X / 2^{k}$.

Though in practice we used this relatively straightforward approach, we also propose another algorithm that, unlike the one described previously, is suited for tabulation of all $k$-untouchable numbers up to a specified bound $X$. Algorithm 2 describes the tabulation procedure. In short, it works as follows: we maintain an array $T[X][K]$ with entries $T[n][k]$ equal to 1 or 0 depending on whether $n$ lies in the range of $s_{k}$ or not. With the help of the Pomerance-Yang algorithm, we initialize a reference table $P$ so that $P[n]=\{m: n=s(m)\}$ for $n \leq X$; and a hash table $Q$ containing ordered triples $(n, m, 1)$ such that $n \leq X$ and $n=s(m)$. We then proceed by calling the recursive subroutine $R(n, m, k)=R(n, m, k, K, X, P, Q, T)$ for each triple $(n, m, k)$ in $Q$ (for the implementation of $R(n, m, k)$ see Algorithm 1). The subroutine $R(n, m, k)$ iterates recursively over all possible preimages of $n$ under $s_{k^{\prime}}$ for $k^{\prime} \in\{k+1, \ldots, K\}$. It returns 0 in one of the following three cases:

(1) if $R$ reaches $m$ such that $n=s_{k^{\prime}}\left(m^{\prime}\right)$ for some odd $m^{\prime}$ and $k^{\prime} \in\{k, k+$ $1, \ldots, K\}$. In this case, by a slightly stronger version of the Goldbach's conjecture described in the previous subsection, it must be the case that $n$ is $k^{\prime}$-touchable for each $k^{\prime} \in\{k, k+1, \ldots, K\}$;

(2) if for some ordered triple $\left(n, m^{\prime}, k^{\prime}\right)$ our function $R$ reaches $k^{\prime}=K$, which means that $n=s_{K}\left(m^{\prime}\right)$, so $n$ is $k^{\prime}$-touchable for each $k^{\prime} \in\{k, k+1, \ldots, K\}$;

(3) there exists $k^{\prime} \in\{k, k+1, \ldots, K\}$ such that the identity $n=s_{k^{\prime}}\left(m^{\prime}\right)$ implies that $m^{\prime}$ is untouchable. In other words, $n$ is $\left(k^{\prime}+1\right)$-untouchable.

\footnotetext{
${ }^{1}$ We have $\# s^{-1}(690100611194)=139$, and this is the maximum over all $\# s^{-1}(n)$ with even $n \leq 2^{40}$. For more details, see the OEIS sequence A283157 Sloane 2017].
} 


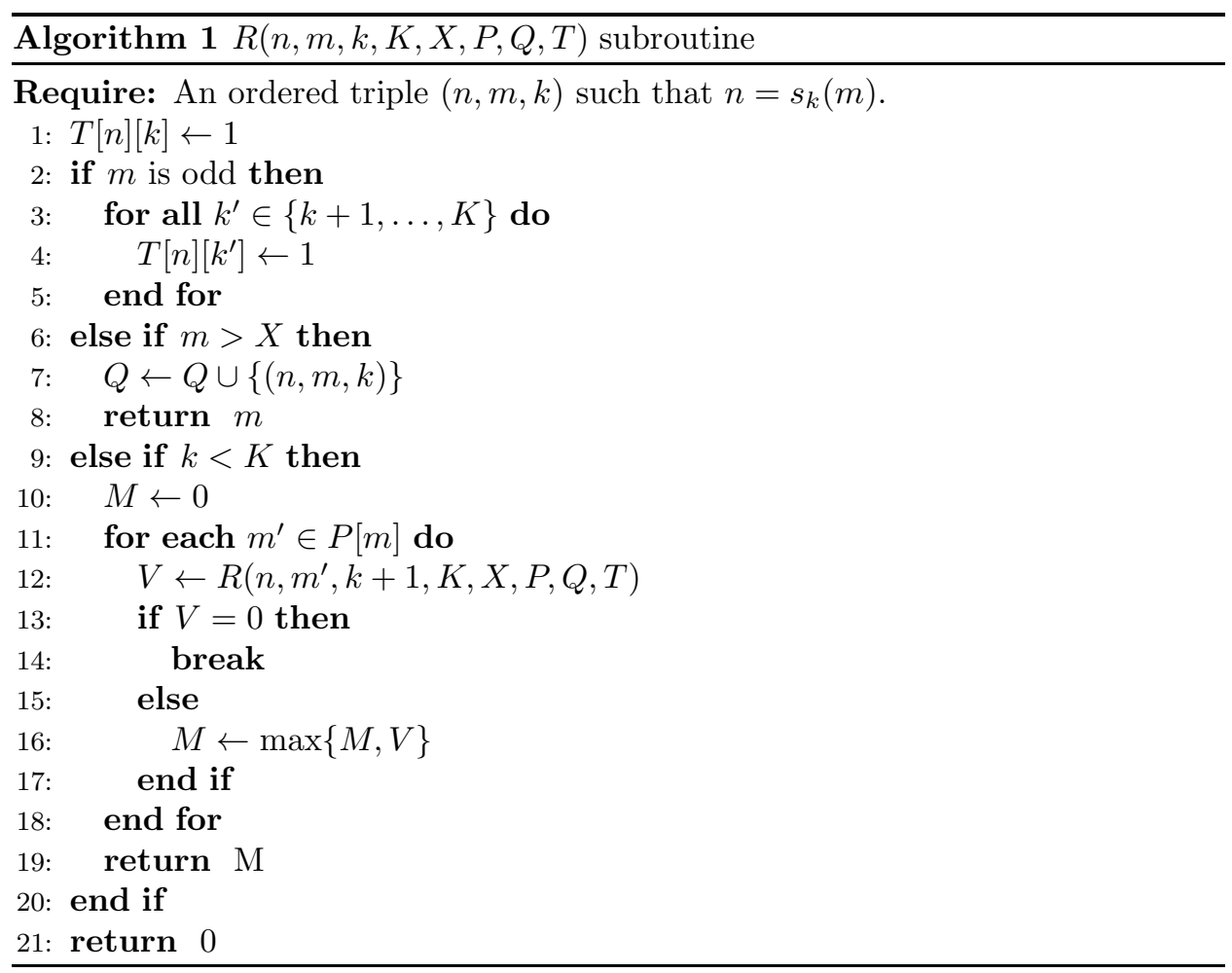

In all of the three cases, upon termination of $R(n, m, k)$ we remove all triples $\left(n^{\prime}, m^{\prime}, k^{\prime}\right)$ in $Q$ with $n^{\prime}=n$, thus indicating that the number $n$ is completely processed and all the values of $T[n]\left[k^{\prime}\right]$ for $k^{\prime} \in\{1,2, \ldots, K\}$ are correct. If, however, the evaluation of $R(n, m, k)$ resulted in some positive integer $M$ instead of 0 , one must face the situation that each recursive call inside $R(n, m, k)$ eventually hits some triple $\left(n, m^{\prime}, k^{\prime}\right)$ with $k^{\prime} \in\{k, k+1, \ldots, K-1\}$ such that $n=s_{k^{\prime}}\left(m^{\prime}\right)$ and $m^{\prime}>X$. While executing, the subroutine $R(n, m, k)$ populates $Q$ with such triples $\left(n, m^{\prime}, k^{\prime}\right)$ and upon termination returns the maximum $M$ over all occurring values of $m^{\prime}$. After that, we call the Pomerance-Yang algorithm again and expand the reference table $P[n]$ up to $n \leq M$. We then call the function $R(n, m, k)$ for each $(n, m, k)$ in $Q$ again, but with $M$ in place of $X$. The call to $R(n, m, k)$ and the further expansion of $P$ are made as many times as needed until $Q$ is empty. At this point, the algorithm terminates.

Let $U_{k}(X)$ denote the total number of $k$-untouchable numbers up to $X$. We computed $U_{k}(X)$ for $X=10^{7}, k=2,3,4,5$, and for $X=10^{8}, k=2,3$. Note that our bound $10^{8}$ is significantly smaller than the bound $2^{40}$, which occurred in the tabulation of untouchable numbers and the values of $\# s^{-1}(n)$. The reason is that in this case we store not just the types of numbers and not just the total number of preimages, but the preimages themselves.

Our choice of parameters is as follows: up to $X=2^{30}$, every even number $n$ has at most 64 preimages, each of which fit into unsigned long ( 8 bytes). Thus the total size of our table of preimages consisting of 4096 files is $\left(2^{30} / 2\right) \cdot 64 \cdot 8$ bytes, which is equal to 256 GB. The tabulation of preimages was carried in parallel, 


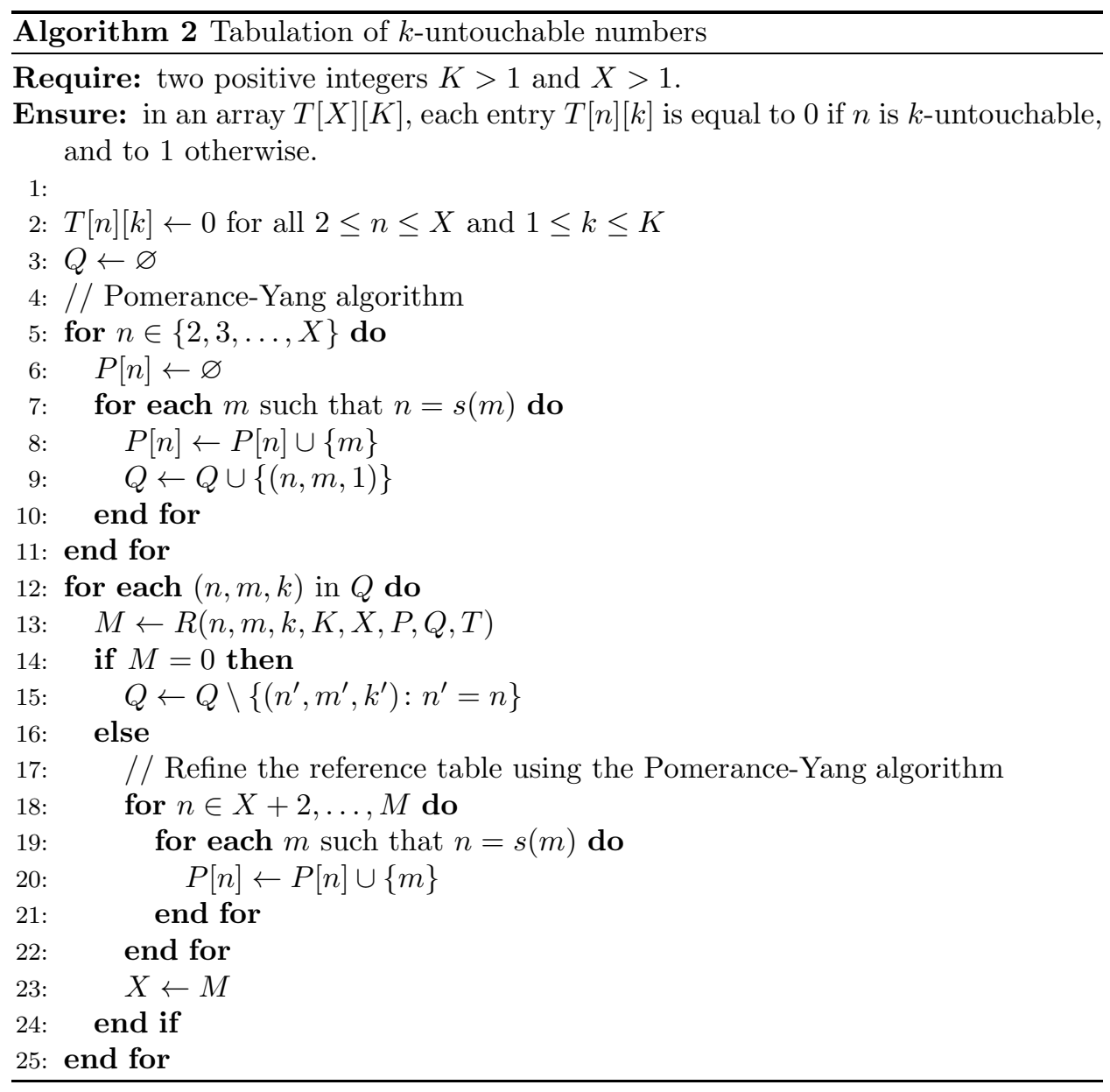

using 64 processors, and the program terminated in $2 \mathrm{~h} 20 \mathrm{~m} 12 \mathrm{~s}$ of real time (the total CPU time is $6 \mathrm{~d} 5 \mathrm{~h} 32 \mathrm{~m} 48 \mathrm{~s}$ ). Then we launched (a simple version of) the tabulation of $k$-untouchable numbers, which was done sequentially. The program for tabulation was launched twice. At the first launch, our program tabulated all $k$-untouchable numbers for $k=1,2,3$ up to $134222590>10^{8}$, and it terminated in $7 \mathrm{~h} 2 \mathrm{~m} 32 \mathrm{~s}$. At the second launch, our program tabulated all $k$-untouchable numbers for $k=1,2,3,4,5$ up to $34100312>10^{7}$, and it terminated in $1 \mathrm{~m} 23 \mathrm{~s}$.

\begin{tabular}{|r||r|r|r|r|r|}
\hline$X$ & $10^{4}$ & $10^{5}$ & $10^{6}$ & $10^{7}$ & $10^{8}$ \\
\hline \hline$U_{1}(X)$ & 1212 & 13863 & 150232 & 1574973 & 16246940 \\
\hline$U_{2}(X)$ & 389 & 4459 & 50824 & 554973 & 5792792 \\
\hline$U_{3}(X)$ & 134 & 1648 & 19628 & 217628 & 2200638 \\
\hline$U_{4}(X)$ & 43 & 594 & 7110 & 79387 & \\
\hline$U_{5}(X)$ & 15 & 208 & 2408 & 27913 & \\
\hline
\end{tabular}

TABLE 3. Counts of $k$-untouchable numbers to $10^{8}$. 
Table 3 contains the counts of $k$-untouchable numbers. Table 4 contains first $10 k$-untouchable numbers for $k=1,2,3,4,5$. The sequences of $k$-untouchable numbers for $k=2,3,4,5$ now appear in the Online Encyclopedia of Integer Sequences Sloane 2017] under identifiers A283152, A284147, A284156 and A284187, respectively.

\begin{tabular}{|r||r|r|r|r|r|r|r|r|r|r|}
\hline$k$ & $a_{1}$ & $a_{2}$ & $a_{3}$ & $a_{4}$ & $a_{5}$ & $a_{6}$ & $a_{7}$ & $a_{8}$ & $a_{9}$ & $a_{10}$ \\
\hline \hline 1 & 2 & 5 & 52 & 88 & 96 & 120 & 124 & 146 & 162 & 188 \\
\hline 2 & 208 & 250 & 362 & 396 & 412 & 428 & 438 & 452 & 478 & 486 \\
\hline 3 & 388 & 606 & 696 & 790 & 918 & 1264 & 1330 & 1344 & 1350 & 1468 \\
\hline 4 & 298 & 1006 & 1016 & 1108 & 1204 & 1502 & 1940 & 2370 & 2770 & 3358 \\
\hline 5 & 838 & 904 & 1970 & 2176 & 3134 & 3562 & 4226 & 5038 & 5580 & 6612 \\
\hline
\end{tabular}

TABLE 4. First $10 k$-untouchable numbers.

Define the quantity

$$
\hat{\lambda}_{k}(X)=\frac{1}{\# C_{k}(X)} \sum_{n \in C_{k}(X)} \log \frac{s(n)}{n},
$$

where

$$
C_{k}(X)=\left\{n \in \mathbb{N}: n \leq X, n \text { is even and in the range of } s_{k}\right\} .
$$

The value of $\hat{\lambda}_{k}(X)$ is equal to the average of $\log (s(n) / n)$ for all even $n \leq X$, with 1-untouchable, 2-untouchable, ..., $k$-untouchable $n$ disregarded. Table 5 depicts the behavior of $\hat{\lambda}_{k}(X)$, and it is clear that removing more and more $k$-untouchable numbers seem to force the average to go down rather than up.

\begin{tabular}{|r||r|r|r|r|r|r|}
\hline$X$ & $k=0$ & $k=1$ & $k=2$ & $k=3$ & $k=4$ & $k=5$ \\
\hline \hline $10^{7}$ & -0.03326 & -0.07370 & -0.08077 & -0.08506 & -0.08618 & -0.08645 \\
\hline $10^{8}$ & -0.03326 & -0.07754 & -0.08982 & -0.09619 & & \\
\hline
\end{tabular}

TABLE 5. Values of $\hat{\lambda}_{k}(X)$.

\section{Discussion}

Although our results to this point seem to favor Catalan-Dickson, they should still be taken with a grain of salt. Even the fact that the Bosma-Kane constant $\mu=0.967 \ldots$ is only slightly less than one suggests that there are many abundant numbers, and it could be the case that some aliquot sequences have a bias towards them. For example, let $S(X)$ and $N S(X)$ be the collections of all square-free and non-square-free even numbers up to $X$, respectively, and consider the quantities

$$
\begin{gathered}
\lambda_{S}(X)=\frac{1}{\# S(X)} \sum_{n \in S(X)} \log \frac{s(n)}{n}, \\
\lambda_{N S}(X)=\frac{1}{\# N S(X)} \sum_{n \in N S(X)} \log \frac{s(n)}{n} .
\end{gathered}
$$


We evaluated $\lambda_{S}\left(10^{r}\right)$ and $\lambda_{N S}\left(10^{r}\right)$ at $r=3,4, \ldots, 10$, and the corresponding values are given in Table 6. Our computations show that $\lambda_{S}(X)$ approaches the limit $-0.3384354384093 \ldots$ proved by Pomerance 2017], but also suggest that the limit

$$
\lim _{X \rightarrow \infty} \lambda_{N S}(X)
$$

exists and, as expected, is close to the limit $0.1747760953325 \ldots$ for integers congruent to 0 mod 4 proved by Pomerance 2017. Thus, most of square-free even numbers are deficient, while most of non-square-free even numbers are abundant. As it was remarked by the second author, the first fact is of no surprise, since the down-driver 2 exactly divides every square-free even number. Also note that in this case the values of $\lambda_{S}(X)$ and $\lambda_{N S}(X)$ are not nearly as close to zero as the value of $\log \mu \approx-0.033$. Hence it could be that some aliquot sequences contain more non-square-free numbers than square-free numbers (such as those with the drivers of Guy and Selfridge [1975]), and because of this they are more likely to go to infinity. The famous aliquot sequences starting at 276, 552, 564, 660 and 966 , so-called Lehmer's Five, - seem to support this heuristic. For example, out of the first 1651 terms of the aliquot sequence $\left\{s_{k}(276)\right\}$ only 596 terms are square-free; for $\left\{s_{k}(552)\right\}$, out of the first 982 terms only 28 are square-free; for $\left\{s_{k}(564)\right\}$, out of the first 3315 terms only 1157 are square-free; for $\left\{s_{k}(660)\right\}$, out of the first 827 terms only 65 are square-free; and for $\left\{s_{k}(966)\right\}$, out of the first 819 terms only 154 are square-free [immermann et al. 2014].

\begin{tabular}{|r||r|r||r|r|}
\hline$X$ & $\# S(X)$ & $\lambda_{S}(X)$ & $\# N S(X)$ & $\lambda_{N S}(X)$ \\
\hline \hline $10^{3}$ & 204 & -0.3342131 & 296 & 0.1701133 \\
\hline $10^{4}$ & 2027 & -0.3379401 & 2973 & 0.1740342 \\
\hline $10^{5}$ & 20267 & -0.3384178 & 29733 & 0.1746938 \\
\hline $10^{6}$ & 202640 & -0.3384442 & 297360 & 0.1747062 \\
\hline $10^{7}$ & 2026416 & -0.3384393 & 2973584 & 0.1747115 \\
\hline $10^{8}$ & 20264234 & -0.3384351 & 29735766 & 0.1747105 \\
\hline $10^{9}$ & 202642377 & -0.3384354 & 297357623 & 0.1747109 \\
\hline $10^{10}$ & 2026423710 & -0.3384354 & 2973576290 & 0.1747109 \\
\hline
\end{tabular}

TABLE 6 . Values of $\lambda_{S}\left(10^{r}\right)$ and $\lambda_{N S}\left(10^{r}\right)$ for $3 \leq r \leq 10$.

\section{Empirical Estimate of Average Growth of Terms}

One possible issue with the data and results described above is that they do not account for the behavior of terms in actual sequences. Thus, our final numerical experiments aim to estimate the geometric mean of $s(n) / n$ empirically.

Drivers and guides, in particular, are two aspects of aliquot sequences that the previous approaches do not take into account. Sometimes a divisor of an integer $n$ may tend to persist throughout repeated applications of the sum-of-proper-divisors function. If this divisor happens to be abundant, then the sequence will tend to increase so long as this divisor remains in subsequent terms. This phenomenon was captured in the notion of guides and drivers, developed by Guy and Selfridge 1975. A guide of $n$ is a divisor of $n$ consisting of the greatest power of 2 that exactly divides $n$, i.e. $2^{a}|| n$ along with any subset of the prime factors of $2^{a+1}-1$. 
If a guide is especially persistent, it is called a driver. Drivers are of the form $2^{a} v$, where $v$ is odd, $v \mid 2^{a+1}-1$, and $2^{a-1} \mid \sigma(v)$. The following theorem enumerates all drivers.

Theorem 1 (Theorem 2 of Guy and Selfridge 1975]). The only drivers are $2,2^{3} \cdot 3$, $2^{3} \cdot 3 \cdot 5,2^{5} \cdot 3 \cdot 7,2^{9} \cdot 3 \cdot 11 \cdot 31$, and the even perfect numbers.

Of these drivers, 2 is a downdriver, while $2 \cdot 3,2^{2} \cdot 7,2^{4} \cdot 31$, and $2^{5} \cdot 3 \cdot 7$ are updrivers. Downdrivers cause an aliquot sequence to decrease, whereas updrivers cause sequences to increase. We focus on these 24 guides: $2^{0}, 2,2 \cdot 3,2^{2}, 2^{2} \cdot 7,2^{3}$, $2^{3} \cdot 3,2^{3} \cdot 5,2^{3} \cdot 3 \cdot 5,2^{4}, 2^{4} \cdot 31,2^{5}, 2^{5} \cdot 3,2^{5} \cdot 7,2^{5} \cdot 3 \cdot 7,2^{6}, 2^{6} \cdot 127,2^{7}, 2^{7} \cdot 3$, $2^{7} \cdot 5,2^{7} \cdot 3 \cdot 5,2^{8}, 2^{9}$, and $2^{\geq 10}$. We say that a guide $g$ is in control of a sequence of terms when $g$ is a guide of said terms.

6.1. Description of Experiments. In his 1976 M.Sc. thesis [Devitt 1976], Devitt presented theoretical and numerical evidence, using a "new method of factoring called Pollard-Rho", that the average order of $s(n) / n$ for $n$ even is greater than 1 . Devitt's method was to construct a Markov chain with states corresponding to the smallest 24 guides. Transition probabilities and averages of $s(n) / n$ for each state were estimated empirically by sampling terms from sequences with various sized terms.

The advantage of this approach is that it captures data on actual sequences, including the effects of drivers and guides. The disadvantage is that it is purely empirical.

We have repeated the experiments that Devitt performed in his thesis, but due to the greater computing power and faster integer factorization algorithms available today, to a much greater range. In addition, we used the geometric mean as opposed to the arithmetic mean used by Devitt.

We collected data from 8000 aliquot sequences: eight sets of 1000 sequences, with each sequence starting at $2^{16+32 n}+2 k$, where $0 \leq n \leq 7$ and $0 \leq k<1000$. Our goal in separating the sequences into these eight stages was to study termination behavior as sequence terms get larger. Each sequence was followed until a term became greater than $2^{288}$. We used the same upper bound for all stages, as this gives every sequence as much of a chance to acquire a down-driver (and perhaps terminate) as we could feasibly enable, and, in addition, more accurately accounts for the observed trend that most sequences tend to increase. We used the Aliqueit software Klasson 2017] to manage the factorization of sequence terms. Aliqueit uses a combination of factorization packages to factor each term, including GMP-ECM, Msieve, Yafu, and GGNFS. We used the ECPP Morain 2017] implementation of the elliptic curve primality proving algorithm Morain 2007 to provide rigorous primality proofs for all probable primes dividing any sequence term.

Data from every sequence term was recorded in matrices as follows. The rows and columns are both indexed by the 25 smallest guides listed in Table 9 , including $2^{0}=1$ as a first column - a sump into which the terminating sequences go. Let $g(n)$ denote the guide of the integer $n$. In the first of a pair of matrices, for each $n$, is accumulated 1 in the $g(s(n))$-th column and $g(n)$-th row. In the second of each pair of matrices is accumulated the amplification, $\log s(n) / n$.

From each pair of matrices we deduced a probability matrix and an amplification matrix. Say that the first has accumulated $t_{i, j}$ terms and the second an amplitude of $a_{i, j}$. Then the probability matrix has entries $p_{i, j}=t_{i, j} / \sum_{i, j} t_{i, j}$. The amplification 
matrix will have entries $A_{i, j}=a_{i, j} / t_{i, j}$. The observed average amplification is

$$
\mathcal{A}=\sum_{i, j} p_{i, j} A_{i, j}
$$

having $\mathcal{A}>0$, i.e., with $e^{\mathcal{A}}>1$ would favor Guy-Selfridge, whereas $\mathcal{A}<0$ would favor Catalan-Dickson. We recorded separate values of $\mathcal{A}$ for each stage, as well as one for all sequences taken together.

In addition to studying the observed amplification values, we again followed Devitt and modeled the sequences using Markov chains in an effort to deduce empirically the expected behavior. This is done by repeatedly squaring the matrix of transition probabilities (a stochastic matrix) until the entries converge. The resulting transition probabilities were used to compute a value of $\mathcal{A}$ that captures more accurately what we would expect on average.

No measures were taken to avoid the effects of tributaries on the calculated values, but we expect the effects of these to be negligible for such large sequence terms.

6.2. Data on Terminating Sequences. In Table 7 we give data on the number of observed occurrences of different types of termination. From all 8000 sequences, we observed that 1544 reached a prime, 6392 passed our limit, and 64 entered a cycle, with a total of 5644436 terms. No new cycles were discovered in the process. Of those sequences that entered a cycle, only 7 were perfect numbers, 47 were amicable numbers, 1 reached a cycle with 5 terms, and 9 reached the cycle with 28 terms. Statistics corresponding to each of the eight stages are included in Table 7 The proportions of sequences which appear not to terminate increase strikingly as the stage increases.

\begin{tabular}{r|cccccccc|c} 
& st 0 & st 1 & st 2 & st 3 & st 4 & st 5 & st 6 & st 7 & Overall \\
start & $2^{16}$ & $2^{48}$ & $2^{80}$ & $2^{112}$ & $2^{144}$ & $2^{176}$ & $2^{208}$ & $2^{240}$ & \\
\hline primes & 709 & 282 & 183 & 122 & 87 & 76 & 43 & 42 & 1544 \\
perfs & 4 & 1 & & & & 1 & 1 & & 7 \\
amics & 26 & 7 & 3 & 6 & 3 & & 1 & 1 & 47 \\
cycles & 6 & 2 & 1 & 1 & & & & & 10 \\
\hline \hline ended & 745 & 292 & 187 & 129 & 90 & 77 & 45 & 43 & 1608 \\
open & 255 & 708 & 813 & 871 & 910 & 923 & 955 & 957 & 6392 \\
\% open & $25.5 \%$ & $70.8 \%$ & $81.3 \%$ & $87.1 \%$ & $91.0 \%$ & $92.3 \%$ & $95.5 \%$ & $95.7 \%$ & $79.9 \%$ \\
\hline
\end{tabular}

TABLe 7. Termination Statistics.

In Table 8, we give percentages of sequences that remained open (i.e. reached our upper bound of $2^{288}$ ) for sequences whose initial terms have various properties. We give the percentages for all sequences, those whose initial terms are $0 \bmod 4$, those whose initial terms are $2 \bmod 4$, those whose initial terms have the down-driver 2 or the guide $2^{3}$, and those whose initial terms are either deficient or abundant. We observe that these properties of the initial terms do have some effect on the eventual fate of a sequence. Pomerance's results Pomerance 2017] show that terms that are $0 \bmod 4$ are expected to increase on average whereas those that are $2 \bmod 4$ decrease, suggesting that sequences whose initial terms have these properties have 


\begin{tabular}{r|cccccccc|c} 
& st 0 & st 1 & st 2 & st 3 & st 4 & st 5 & st 6 & st 7 & Overall \\
start & $2^{16}$ & $2^{48}$ & $2^{80}$ & $2^{112}$ & $2^{144}$ & $2^{176}$ & $2^{208}$ & $2^{240}$ & \\
\hline all & $25.5 \%$ & $70.8 \%$ & $81.3 \%$ & $87.1 \%$ & $91.0 \%$ & $92.3 \%$ & $95.5 \%$ & $95.7 \%$ & $79.9 \%$ \\
0 mod 4 & $26.6 \%$ & $71.2 \%$ & $83.0 \%$ & $88.4 \%$ & $91.8 \%$ & $93.0 \%$ & $96.2 \%$ & $96.6 \%$ & $80.9 \%$ \\
abundant & $38.8 \%$ & $76.2 \%$ & $86.4 \%$ & $90.7 \%$ & $94.4 \%$ & $95.0 \%$ & $97.3 \%$ & $97.6 \%$ & $84.6 \%$ \\
2 mod 4 & $24.4 \%$ & $70.2 \%$ & $79.6 \%$ & $85.8 \%$ & $90.2 \%$ & $91.6 \%$ & $94.8 \%$ & $94.8 \%$ & $79.0 \%$ \\
deficient & $12.6 \%$ & $65.6 \%$ & $76.4 \%$ & $83.7 \%$ & $87.6 \%$ & $89.7 \%$ & $93.7 \%$ & $93.8 \%$ & $75.3 \%$ \\
2 or $2^{3}$ & $9.5 \%$ & $63.9 \%$ & $73.4 \%$ & $82.0 \%$ & $87.2 \%$ & $89.0 \%$ & $93.0 \%$ & $93.5 \%$ & $73.9 \%$ \\
\hline
\end{tabular}

TABle 8. Percentage of "Open" Sequences for Initial Terms with Different Properties.

a better chance to increase overall or terminate, respectively. Similarly, sequences that start with a deficient number begin by decreasing, and hence may have a better chance to terminate, whereas those that begin with an abundant number should tend to increase more often. Finally, sequences whose initial terms have as a guide the down-driver 2 or $2^{3}$ should have an even better chance of terminating, as these terms should drive the sequence more persistently down. Our data shows that this is in fact what happens in practice. However, the effect of these properties of the initial term diminishes as the starting term itself increases.

6.3. Data on Non-Terminating Sequences. In Table 9, we list the guides that were active for $n$ when $s(n)$ breached $2^{288}$ for the 6392 sequences that did not terminate.

None of the terms here having $2^{9}$ as a guide contained the driver $2^{9} \cdot 3 \cdot 11 \cdot 31$. It seems that a good portion of the terms are under the control of an up-driver. There are also surprisingly many terms that have $2^{2}$ as a guide. Since Pomerance's result Pomerance 2017] shows that the geometric mean of $\log (s(n) / n)$ for $n$ divisible by 4 is greater than zero, this is in fact further evidence in support of Guy-Selfridge.

6.4. Average Amplification. For each guide that we considered, we recorded in Table 10 the number of occurrences, the number of "runs" (consecutive sequence terms of length $\geq 2$ with that guide), the average length of a run, the logarithm of the average amplification over all terms with that guide, and the average amplification over each run with that guide. The initial term of each of the 8000 sequences is excluded in these counts, as amplification is not defined for the initial terms that do not have a predecessor. For the even guides, we also recorded the expected number of occurrences of each guide, assuming that divisibility properties of the sequence terms are the same as for random integers. For example, of the 5632116 even sequence terms we would expect one third of them, 1877372, to contain a factor 2 but no higher power of 2 .

Although the down-driver 2 was the most frequently occurring, the up-drivers collectively occurred much more frequently than the down-drivers. Notice that the down-driver 2 occurs only on about $2 / 3$ of the number of occasions than would be expected, while the up-drivers $2^{2} \cdot 7,2^{3} \cdot 3 \cdot 5,2^{4} \cdot 31,2^{5} \cdot 3 \cdot 7,2^{6} \cdot 127, \ldots$ all occur several times more frequently than expected, suggesting that on average we might expect terms in aliquot sequences to increase rather than decrease. 


\begin{tabular}{r|rl} 
Guide & \# Active & Driver Type \\
\hline $2^{0}$ & 0 & down-driver \\
$2^{1}$ & 0 & down-driver \\
$2 \cdot 3$ & 1124 & up-driver \\
$2^{2}$ & 374 & \\
$2^{2} \cdot 7$ & 1620 & up-driver \\
$2^{3}$ & 8 & \\
$2^{3} \cdot 3$ & 584 & \\
$2^{3} \cdot 5$ & 75 & \\
$2^{3} \cdot 3 \cdot 5$ & 773 & up-driver \\
$2^{4}$ & 386 & \\
$2^{4} \cdot 31$ & 531 & up-driver \\
$2^{5}$ & 12 & \\
$2^{5} \cdot 3$ & 158 & \\
$2^{5} \cdot 7$ & 52 & \\
$2^{5} \cdot 3 \cdot 7$ & 278 & up-driver \\
$2^{6}$ & 126 & \\
$2^{6} \cdot 127$ & 143 & up-driver \\
$2^{7}$ & 2 & \\
$2^{7} \cdot 3$ & 49 & \\
$2^{7} \cdot 5$ & 15 & \\
$2^{7} \cdot 3 \cdot 5$ & 24 & \\
$2^{8}$ & 33 & \\
$2^{9}$ & 12 & \\
$2^{10}$ & 0 & up-driver \\
$2^{9} \cdot 3 \cdot 11 \cdot 31$ & 13 & \\
\hline & &
\end{tabular}

TABLE 9. Active guides for non-terminating sequences.

Following the procedure described in Section 6.1, we computed the average amplification per term and the expected amplification via Markov chain analysis, individually for experiments at each of the eight stages and for all sequences collectively. The results are given in Table 11. In all cases, both the observed average per term and the expected average are greater than zero, giving empirical evidence that terms in aliquot sequences grow on average. The fact that the majority of sequence terms come from open sequences that are increasing certainly contributes to these statistics being greater than zero, but this is, based on our observations, an accurate assessment of the average behavior of a sequence. Overall, our results indicate that the geometric mean of $s(n) / n$ is approximately $e^{0.125} \approx 1.133$, lending support to the Guy-Selfridge conjecture.

In Table 12, we give the observed and expected averages when restricting to initial terms with particular properties. These data are computed over all sequences considered from all 8 stages. As above, we expect that the initial term will have some influence over the average amplifications; initial terms that are $0 \bmod 4$ or abundant should cause a slight increase, whereas initial terms that are $2 \bmod 4$, deficient, or starting with a down-driver should cause a decrease, with the largest occurring for the down-driver case. This is exactly what we observed. However, it is noteworthy that the average amplification, in all cases, is still greater than zero, 


\begin{tabular}{rrrrrr} 
Guide & $\begin{array}{r}\text { Times } \\
\text { Seen }\end{array}$ & $\begin{array}{r}\text { Expected } \\
\text { Number }\end{array}$ & $\begin{array}{r}\text { Runs } \\
\text { Average } \\
\text { Length }\end{array}$ & $\begin{array}{r}\text { Average } \\
\text { Amplification }\end{array}$ \\
\hline $2^{0}$ & 4320 & - & 1777 & 2.431 & -0.777 \\
2 & 1288576 & 1877372 & 42353 & 30.488 & -0.470 \\
$2 \cdot 3$ & 746474 & 938686 & 5080 & 147.207 & 0.226 \\
$2^{2}$ & 858815 & 1206882 & 58467 & 14.718 & 0.069 \\
$2^{2} \cdot 7$ & 937561 & 201147 & 9369 & 100.101 & 0.333 \\
$2^{3}$ & 269723 & 375474 & 45753 & 5.907 & -0.032 \\
$2^{3} \cdot 3$ & 213178 & 187737 & 12274 & 17.390 & 0.536 \\
$2^{3} \cdot 5$ & 124637 & 93869 & 13619 & 9.162 & 0.322 \\
$2^{3} \cdot 3 \cdot 5$ & 139573 & 46934 & 3177 & 43.953 & 0.802 \\
$2^{4}$ & 316435 & 340652 & 65368 & 4.848 & 0.303 \\
$2^{4} \cdot 31$ & 257444 & 11355 & 2051 & 125.529 & 0.362 \\
$2^{5}$ & 83619 & 100574 & 32961 & 2.541 & 0.107 \\
$2^{5} \cdot 3$ & 60418 & 50287 & 11673 & 5.182 & 0.640 \\
$2^{5} \cdot 7$ & 40648 & 16762 & 4963 & 8.195 & 0.345 \\
$2^{5} \cdot 3 \cdot 7$ & 54003 & 8381 & 1630 & 33.139 & 0.813 \\
$2^{6}$ & 87858 & 87309 & 35140 & 2.504 & 0.357 \\
$2^{6} \cdot 127$ & 57622 & 693 & 306 & 188.307 & 0.348 \\
$2^{7}$ & 20953 & 23467 & 12059 & 1.740 & 0.096 \\
$2^{7} \cdot 3$ & 14195 & 11734 & 5466 & 2.600 & 0.631 \\
$2^{7} \cdot 5$ & 6941 & 5867 & 3410 & 2.038 & 0.431 \\
$2^{7} \cdot 3 \cdot 5$ & 4511 & 2933 & 1407 & 3.212 & 0.909 \\
$2^{8}$ & 23925 & 22000 & 13507 & 1.774 & 0.386 \\
$2^{9}$ & 12880 & 11000 & 7831 & 1.647 & 0.399 \\
$2^{\geq 10}$ & 12127 & 11000 & 7852 & 1.545 & 0.391 \\
\hline & & & & &
\end{tabular}

TABLE 10. Data on occurrences, runs, and amplification for each guide.

\begin{tabular}{l|llllllll|l} 
Amplification & st 0 & st 1 & st 2 & st 3 & st 4 & st 5 & st 6 & st 7 & Overall
\end{tabular}

\begin{tabular}{r|cccccccc|c}
\hline average & 0.135 & 0.127 & 0.117 & 0.111 & 0.102 & 0.086 & 0.073 & 0.048 & 0.101 \\
expected & 0.127 & 0.139 & 0.136 & 0.135 & 0.132 & 0.121 & 0.116 & 0.103 & 0.125 \\
\hline
\end{tabular}

TABle 11. Average Amplification $\log (s(n) / n)$ Statistics.

\begin{tabular}{r|cccccc} 
Amplification & all & $0 \bmod 4$ & abundant & $2 \bmod 4$ & deficient & 2 or $2^{3}$ \\
\hline average & 0.101 & 0.106 & 0.122 & 0.097 & 0.084 & 0.080 \\
expected & 0.125 & 0.127 & 0.131 & 0.124 & 0.121 & 0.119 \\
\hline
\end{tabular}

TABLE 12. Average Amplification $\log (s(n) / n)$ Statistics for Varying Initial Terms.

indicating that even when considering only sequences that start by decreasing, on average the terms will tend to increase, and lending even more support to the Guy-Selfridge conjecture. 


\section{Conclusions}

Our results are mixed, and do not lead to a definitive conclusion. Most of the approaches related to analytic methods suggest that terms in aliquot sequences tend to decrease on average, except when restricting to non-square-free even values. On the other hand approximating the average growth of terms experimentally suggests that terms tend to increase. We hypothesize that the discrepancy is due to the effect of guides and drivers, which are taken into account in the pure numerical estimates but not in those inspired by recent analytic results. Extensions of the

analytic methods that account for their effect, further extending the recent work of Pomerance [2017] would thus be a natural avenue for future work.

\section{ACKNowledgements}

The authors wish to thank Carl Pomerance and the anonymous referee for many helpful comments and suggestions.

\section{REFERENCES}

Bosma, W. and Kane, B. (2012). The aliquot constant. The Quarterly Journal of Mathematics, 63(2):309 - 323.

Catalan, E. (1888). Propositions et questions diverses. Bull. Soc. Math. France, $16: 128-129$.

Devitt, J. S. (1976). Aliquot sequences. Master's thesis, University of Calgary.

Dickson, L. E. (1913). Theorems and tables on the sums of divisors of a number. Quart. J. Math., 44:264- 296.

Erdős, P. (1973). Über die Zahlen der Form $\sigma(n)-n$ und $n-\phi(n)$. Elem. Math., $11: 83-86$.

Erdős, P. (1976). On asymptotic properties of aliquot sequences. Mathematics of Computation, 30(135):641 - 645 .

Guy, R. K. and Selfridge, J. L. (1975). What drives an aliquot sequence? Mathematics of Computation, 29(129):101 - 107.

Klasson, M. (2017). Aliqueit. Available at http://mklasson.com/aliquot.php Last accessed: 2017-10-19.

Luca, F. and Pomerance, C. (2015). The range of the sum-of-proper-divisors function. Acta Arithmetica, 168:187 - 199.

Moews, D. and Moews, P. C. (1991). A search for aliquot cycles below $10^{10}$. Mathematics of Computation, 57(196):849 - 855.

Morain, F. (2007). Implementing the asymptotically fast version of the elliptic curve primality proving algorithm. Math. Comp., 76:493-505.

Morain, F. (2017). The ECPP home page. Available at http://www.lix.polytechnique.fr/ morain/Prgms/ecpp.english.html. Last accessed: 2017-11-06.

Oliveira e Silva, T. (2013). Goldbach conjecture verification. Available at http://sweet.ua.pt/tos/goldbach.html. Last accessed: 2017-08-16.

Pollack, P. and Pomerance, C. (2016). Some problems of Erdos on the sum-ofdivisors function. Transactions of the American Mathematical Society, Series B, $3: 1-26$.

Pollack, P., Pomerance, C., and Thompson, L. (2018). Divisor-sum fibers. Mathematika, 64(2):330 - 342 . 
Pomerance, C. (2017). The aliquot constant, after Bosma and Kane. Unpublished manuscript. Available online at https://www.math.dartmouth. edu/ carlp/aliconstantpaper2.pdf.

Pomerance, C. (2018). The first function and its iterates. In Butler, S., Cooper, J., and Hurlbert, G., editors, Connections in Discrete Mathematics: A Celebration of the Work of Ron Graham, pages 125-138. Cambridge University Press.

Pomerance, C. and Yang, H.-S. (2014). Variant of a theorem of Erdös on the sum-of-proper-divisors function. Mathematics of Computation, 83:1903-1913.

Sloane, N. J. A. (2017). The On-Line Encyclopedia of Integer Sequences. Published electronically at https://oeis.org. Last accessed: 2017-10-06.

WestGrid (2017). Hungabee specification. Available at https://www.westgrid.ca/support/systems/Hungabee. Last accessed: 2017-08-16.

Zimmermann, P., Howell, J., Hoogendoorn, S., and Creyaufmueller, W. (2014). Extensive computations of first five openend aliquot sequences. Available at http://www.aliquot.de/lehmer.html. Last accessed: 2017-08-30.

University of Calgary, 2500 University Drive NW, Calgary, Alberta, Canada T2N $1 \mathrm{~N} 4$

Email address: kchum@ucalgary.ca

University of Calgary, 2500 University Drive NW, Calgary, Alberta, Canada T2N $1 \mathrm{~N} 4$

Email address: rkg@cpsc.ucalgary.ca

University of Calgary, 2500 University Drive NW, Calgary, Alberta, Canada T2N $1 \mathrm{~N} 4$

Email address: jacobs@cpsc.ucalgary.ca

University of Waterloo, 200 University Ave W, Waterloo, Ontario, Canada N2L 3G1

Email address: amosunov@uwaterloo.ca 